\section{TUMOR-SPECIFIC REACTIVITY AND EFFECTOR FUNCTION OF CHIMERIC ANTIGEN RECEPTOR ENGINEERED MACROPHAGES TARGETING MUC1}

Seth Eisenberg*, Amy Powers, Jason Lohmueller, James Luketich, Rajeev Dhupar, Adam Soloff. University of Pittsburgh School of Medicine, Pittsburgh, PA, USA

Background Chimeric antigen receptors (CAR) have demonstrated remarkable efficacy in licensing $\mathrm{T}$ cells for antitumor responses against hematopoietic malignancies but have had limited success against solid tumors. Macrophages, both archetypic phagocytes and professional antigen presenting cells, may exert profound effector functions which complement adaptive cellular immunity. ${ }^{1}$ Recently, it was shown that human macrophages engineered to express CARs (CAR-Ms) demonstrated antigen-specific phagocytosis, inhibited solid xenograph tumors, and induced an inflammatory tumor microenvironment boosting antitumor $\mathrm{T}$ cell responses. ${ }^{2}$ Kimura et al. previously completed the first prophylactic cancer vaccine trial based on a non-viral antigen, tumor-associated hypoglycosylated Mucin 1 (MUC1). ${ }^{3}$ A panel of fully-human affinity-matured MUC1-specific antibodies raised in healthy subjects following immunization was identified from these patients. ${ }^{4}$ Using these MUC1specific $s c F v$ domains for CAR generation, we have now engineered MUC1-targeting CAR-Ms that may potentially possess reduced off-target specificities.

Methods Lentiviral CAR expression vectors containing the $\mathrm{scFv}$ domains of three unique hypoglycosylated MUC1-specific antibodies or a CD20-specific antibody, the CD3zeta signaling domain, and CD28 and OX40 co-stimulatory domains were constructed. The human monocyte/macrophage U937, SC, and THP-1 lines were stably transduced and flow-sort purified to generate MUC1- or CD20-specific CAR-Ms. CAR-Ms were differentiated into macrophages via 48 hour PMA treatment, and subsequently evaluated for antigen-specific function against MUC1- and/or CD20-expressing K562, ZR-75-1, and Raji cells or cancer cells isolated from solid lung tumors or malignant pleural effusions. CAR-M phenotype was evaluated by flow cytometry following in vitro differentiation and polarization with conventional 'M1' and 'M2' stimuli. Phagocytosis and lysosomal processing of phagocytosed cargo were evaluated by fluorescence microscopy of GFP/CellTrace labeled targets or detection of $\mathrm{pH}$-sensitive pHrodo expression following CAR-M and tumor cell co-culture, respectively. Antigenspecific cytokine production was determined via cytometric bead array following co-culture of CAR-Ms with MUC1- or CD20-expressing tumor cells or 100 mer MUC1 peptide.

Results Differentiated CAR-Ms possessed an inflammatory phenotype expressing IL-8 and CD86 which was further enhanced by IFNgamma or LPS treatment and was resistant to 'M2' polarization with conventional stimuli. CAR-Ms exhibited phagocytosis and subsequent lysosomal processing in an antigen-specific manner, with minimal reactivity against tumor cell targets in the absence of the corresponding MUC1 or CD20 antigen. MUC1-specific CAR-Ms stimulated with MUC1 peptide or MUC1+ tumor cells secreted robust levels of proinflammatory IL-8, TNFa, and IL-1beta, but not immunosuppressive IL-10.

Conclusions MUC1-targeting CAR-Ms exert potent tumorrestricted effector function in vitro and may provide a novel treatment strategy either alone or in potential synergistic combination with $\mathrm{T}$ cell-mediated immunotherapies.

Acknowledgements The authors would like to thank Dr. Olivera J. Finn for generously providing reagents and guidance and Dr. Michael T. Lotze for his mentorship. This study was supported by funding from the University of Pittsburgh's Department of Cardiothoracic Surgery to ACS and RD.

\section{REFERENCES}

1. Williams CB, Yeh ES, Soloff AC. Tumor-associated macrophages: unwitting accomplices in breast cancer malignancy. Npj Breast Cancer [Internet]. Breast Cancer Research Foundation/Macmillan Publishers Limited; 2016;2:15025. Available from: http://dx.doi.org/10.1038/npjbcancer.2015.25

2. Klichinsky M, Ruella M, Shestova O, Lu XM, Best A, Zeeman M, et al. Human chimeric antigen receptor macrophages for cancer immunotherapy. Nat Biotechnol 2020:38:947-53.

3. Kimura T, McKolanis JR, Dzubinski LA, Islam K, Potter DM, Salazar AM, et al. MUC1 Vaccine for Individuals with Advanced Adenoma of the Colon: A Cancer Immunoprevention Feasibility Study. Cancer Prev Res [Internet] 2013;6:18-26. Available from: http://cancerpreventionresearch.aacrjournals.org/content/6/1/18. abstract

4. Lohmueller JJ, Sato S, Popova L, Chu IM, Tucker MA, Barberena R, et al. Antibodies elicited by the first non-viral prophylactic cancer vaccine show tumor-specificity and immunotherapeutic potential. Sci Rep 2016;6:31740.

Ethics Approval The study was approved by the University of Pittsburgh's Institutional Review Board approval number CR19120172-005.

http://dx.doi.org/10.1136/jitc-2021-SITC2021.112 